\title{
Scale transition and enforcement of RVE boundary conditions in second-order computational homogenization
}

\author{
Łukasz Kaczmarczyk, Chris J. Pearce*,† and Nenad Bićanić \\ Department of Civil Engineering, University of Glasgow, Rankine Building, Glasgow G12 8LT, U.K.
}

\begin{abstract}
SUMMARY
Formulation of the scale transition equations coupling the microscopic and macroscopic variables in the second-order computational homogenization of heterogeneous materials and the enforcement of generalized boundary conditions for the representative volume element (RVE) are considered. The proposed formulation builds on current approaches by allowing any type of RVE boundary conditions (e.g. displacement, traction, periodic) and arbitrary shapes of RVE to be applied in a unified manner. The formulation offers a useful geometric interpretation for the assumptions associated with the microstructural displacement fluctuation field within the RVE, which is here extended to second-order computational homogenization. A unified approach to the enforcement of the boundary conditions has been undertaken using multiple constraint projection matrices. The results of an illustrative shear layer model problem indicate that the displacement and traction RVE boundary conditions provide the upper and lower bounds of the response determined via second-order computational homogenization, and the solution associated with the periodic RVE boundary conditions lies between them. Copyright 2007 John Wiley \& Sons, Ltd.
\end{abstract}

Received 10 May 2007; Revised 31 July 2007; Accepted 14 August 2007

KEY WORDS: solids; finite element method; multiscale; second-order continuum; computational homogenization; scale transition

\section{INTRODUCTION}

A wide range of manufactured, as well as natural, materials are heterogeneous at a certain scale of observation. In terms of modelling, the macroscopic (equivalent) properties of a heterogeneous material are required to represent the essence of the microstructural response and they must be

\footnotetext{
*Correspondence to: Chris J. Pearce, Department of Civil Engineering, University of Glasgow, Rankine Building, Glasgow G12 8LT, U.K.

${ }^{\dagger}$ E-mail: c.pearce@civil.gla.ac.uk

Contract/grant sponsor: U.K. Engineering and Physical Sciences Research Council; contract/grant number: $\mathrm{EP} / \mathrm{D} 500273$

Contract/grant sponsor: Polish Ministry of Scientific Research and Information Technology Society; contract/grant number: 4 T07E060 29
}

Copyright 2007 John Wiley \& Sons, Ltd. 


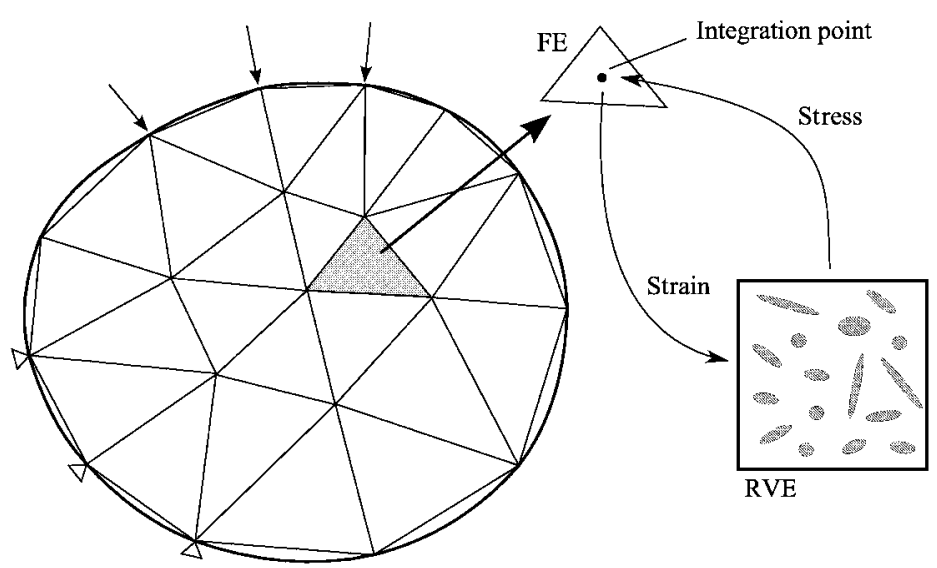

Figure 1. Illustration of computational homogenization scheme.

independent of macrostructural loads and geometry. Furthermore, the transition of properties and variables between the micro- and macro-levels must be consistent with the basic principles of continuum mechanics, i.e. they are subject to the principles of conservation of mass, momentum, energy, and the second law of thermodynamics.

A comprehensive review of the overall properties of heterogeneous materials is provided in [1]. Traditionally, equivalent material properties have been obtained by means of analytical or semi-analytical homogenization techniques. In recent years, a promising alternative approach has been developed, i.e. computational homogenization [2]. This micro-macro modelling procedure does not lead to closed-form constitutive relations but determines the stress-strain relationship at a selected macro-level point to which a detailed microstructure is attributed (representative volume element (RVE), Figure 1). Such an approach does not require the constitutive response on the macro-level to be known a priori and enables the incorporation of both non-linear geometric and material behaviour $[2,3]$. Moreover, computational homogenization is possible for any discretization technique in both space and time, although the finite element method has traditionally been adopted for quasi-static problems. Kouznetsova [2] presented a second-order computational homogenization framework, whereby all microstructural constituents are treated as classical continua and described by classical equilibrium and constitutive equations, while periodic boundary conditions were applied to the RVE. Here, Kouznetsova's second-order scheme is extended to encompass not only periodic-type boundary conditions for the RVE but also traction and displacement boundary conditions in a generalized unified manner.

Multi-scale models are constructed using three main ingredients (Figure 1) [4]:

1. modelling of mechanical behaviour at the microscale (RVE);

2. a downscaling rule that determines the microscale boundary value problem for the RVE, given the macroscopic deformation measures;

3. an upscaling rule for the macroscopic stress measures, given the micromechanical stress state.

In Section 2 this paper briefly reviews the formulation of the second-order continua. Section 3 focusses on the transition between the macro and micro scales and in particular the imposition 
of boundary conditions applied to the RVE and the relationship between the macroscopic strains (macrostrains) and the macroscopic stresses (macrostresses) expressed in terms of the displacements and traction forces on the RVE boundary. Section 4 proposes a novel approach to treat RVE boundary conditions in the context of computational homogenization by employing a general multiple constraints approach [5] for the imposition of essential boundary conditions, while the macroscopic stresses and tangent moduli are defined in terms of discrete forces and stiffness properties of the RVE. In Section 5, a numerical example of a boundary shear layer problem concerning a porous material is considered.

\section{SECOND-ORDER CONTINUA}

In the classical (first-order) continuum theory, the material behaviour is characterized in terms of pointwise stresses and strains and exhibits no size effect. This paper, however, focusses on problems for which the macroscopic stress-strain behaviour also depends on the characteristic size of the microstructure such that higher-order effects are present. Therefore, the assumption of locality adopted in classical continuum theory, whereby the stresses at a point depend only on the strains (and other state variables) at the same point, is no longer adequate and the material behaviour at a given point also depends on the deformation in the neighbourhood of that point. Toupin [6] and Mindlin [7] proposed a general strain gradient (non-local) continuum theory which will be adopted here for the macroscale level (summarized below for the sake of completeness).

Quasi-static geometrically linear problems without thermal effects are considered. It is assumed that the local internal work at the macroscale can be expressed as

$$
W=\boldsymbol{\sigma}: \varepsilon+\tau: \eta
$$

where the first-order stress $\boldsymbol{\sigma}$ and the second-order stress $\tau$ are work conjugate to the first-order strain $\boldsymbol{\varepsilon}=\operatorname{sym}[\operatorname{grad}[\mathbf{u}]]$ and second-order strain $\boldsymbol{\eta}=\operatorname{grad}[\operatorname{grad}[\mathbf{u}]]$ respectively, where $\mathbf{u}$ is the displacement field.

Following [6], for a body of volume $V$ and surface $\Gamma$ with normal $\mathbf{n}$, the boundary value problem for a second-order continuum is expressed by the following equilibrium equation:

$$
\operatorname{div}[\widehat{\boldsymbol{\sigma}}]+\mathbf{f}=\mathbf{0} \text { in } V
$$

where the real stress $\widehat{\boldsymbol{\sigma}}$ is defined as

$$
\widehat{\boldsymbol{\sigma}}=\boldsymbol{\sigma}-\operatorname{div}[\tau]
$$

In addition, on the part of the surface $\Gamma_{t}$ where tractions $\mathbf{t}$ are prescribed, it holds that

$$
\mathbf{n} \cdot \widehat{\boldsymbol{\sigma}}+\mathbf{D}^{\mathrm{s}}[\mathbf{n}] \otimes \mathbf{n} \cdot \mathbf{n} \cdot \tau-\mathbf{D}^{\mathrm{s}}[\mathbf{n} \cdot \tau]-\mathbf{t}=\mathbf{0} \quad \text { on } \Gamma_{t}
$$

where $\mathbf{n}$ is the normal unit vector of surface $\Gamma, \mathbf{D}^{\mathrm{s}}$ is the surface-gradient operator defined as

$$
\mathbf{D}^{\mathrm{s}}[.]=(\mathbf{I}-\mathbf{n} \otimes \mathbf{n}): \operatorname{grad}[.]
$$

and $\mathbf{I}$ is the second-order identity tensor. On the part of the surface $\Gamma_{\mathrm{s}}$ where double tractions $\mathbf{S}$ are prescribed, it follows that

$$
\mathbf{n} \cdot \mathbf{n} \cdot \tau-\mathbf{s}=\mathbf{0} \quad \text { on } \Gamma_{\mathrm{s}}
$$


On the remaining part of the surface $\Gamma_{u}$ the displacements and normal gradients of displacement should be prescribed. The surface $\Gamma$ is assumed to be smooth.

When undertaking conventional displacement-based finite element analysis of second-order (i.e. strain gradient) continua, the interpolation of displacements should exhibit at least $C^{1}$ continuity. However, due to the complex formulation and practical difficulties associated with the implementation of such elements, the use of $C^{0}$ continuous elements is preferred. Thus, a formulation based on Shu et al. [8] has been utilized, whereby relaxed displacement gradients are introduced as additional degrees of freedom, together with an additional kinematic constraint to enforce equality between these additional degrees of freedom and the displacement gradients. For practical computations involving second-order continua in two dimensions, Shu et al. [8] recommend the use of the nine-node isoparametric quadrilateral element (QU34L4). Such an element is stable and it was found to work well for nearly incompressible solids.

\section{SCALE TRANSITION}

Attention is now focussed on the transition strategy for the strains and strain gradients from the macroscale to the discretized microstructure. The proposed method is used to couple two different continua: a classical (first-order) continuum at the microscale (RVE), and a higher- (second) order continuum at the macroscale. The boundary conditions for the RVE enforce, in an average sense, the deformation of the RVE according to a given macroscopic strain and strain gradient.

Averaging theorems implicit in the first-order computational homogenization scheme have a suitable geometric interpretation-i.e. that both the area and the first moment of area of the deformed RVE (with respect to an arbitrary axis) defined in terms of microscopic displacements are equal to the area and first moment of area of the RVE expressed in terms of macroscopic deformation measures. This assumption consequently places a restriction on the microscopic displacement field which, together with the boundary conditions, leads to a well-posed boundary value problem for deformation of the RVE. Here it is proposed to extend this concept to second-order computational homogenization, i.e. to include an additional assumption that the second moment of area of the deformed RVE given in terms of microscopic displacements is equal to the second moment of area of the RVE expressed in terms of macroscopic deformation measures.

For the first-order computational homogenization theory, it has been concluded by a number of authors (for example, [9]) that periodic boundary conditions applied to the RVE lead to the best estimate of the overall response, as compared with the uniform traction boundary conditions (lowerbound estimation) and uniform displacement boundary conditions (upper-bound estimation). These findings have tacitly been extended to the case of second-order computational homogenization [2] such that only periodic boundary conditions have been considered and the formulation restricted to square RVEs. The present work goes beyond that, as it considers a unified way of enforcing generalized RVE boundary conditions, which can be applied to arbitrarily shaped RVEs.

\subsection{Downscaling}

The displacement field within the RVE is expressed by a truncated Taylor's series expansion of the displacement vector about the geometric centre of the RVE:

$$
\mathbf{u}(\mathbf{X}, \mathbf{x})=\mathbf{u}^{0}(\mathbf{X})+\mathbf{x} \cdot \overline{\boldsymbol{\varepsilon}}(\mathbf{X})+\frac{1}{2} \mathbf{x} \otimes \mathbf{x}: \overline{\boldsymbol{\eta}}(\mathbf{X})+\mathbf{r}(\mathbf{X}, \mathbf{x})
$$


where $\mathbf{u}^{0}(\mathbf{X})$ is the displacement of the macroscopic point to which the RVE is assigned, $\overline{\boldsymbol{\varepsilon}}$ is the macrostrain tensor, $\overline{\boldsymbol{\eta}}$ is the second-order macrostrain tensor, $\mathbf{x}$ is the fast varying local RVE spatial coordinate, and $\mathbf{X}$ is the slow varying macroscopic spatial coordinate. The additional term $\mathbf{r}$ represents the microstructural displacement fluctuation field, which has been added to account for the microscale contribution to the displacement field.

In the following, the symmetric operator is dropped since it has been assumed, for simplicity, that the rotation of the RVE is negligible. Taking the spatial derivative of (7) leads to the microscopic strain (microstrain) field within the RVE

$$
\boldsymbol{\varepsilon}=\operatorname{grad}[\mathbf{u}]=\overline{\boldsymbol{\varepsilon}}+\mathbf{x} \cdot \overline{\boldsymbol{\eta}}+\operatorname{grad}[\mathbf{r}]
$$

The volume average of the microstrain field yields

$$
\frac{1}{V} \int_{V} \boldsymbol{\varepsilon} \mathrm{d} V=\overline{\boldsymbol{\varepsilon}}+\frac{1}{V} \int_{V} \mathbf{x} \mathrm{d} V \cdot \overline{\boldsymbol{\eta}}+\frac{1}{V} \int_{V} \operatorname{grad}[\mathbf{r}] \mathrm{d} V
$$

In the case that the RVE is centred on the corresponding macroscopic point (i.e. that the local RVE spatial coordinates have their origin at the geometric centre of the RVE), the second term on the right-hand side of (9) is automatically zero (first moment of area about the centroid); thus, the microscale fields $(\mathbf{r}, \boldsymbol{\varepsilon})$ can be related to the macrostrain $\overline{\boldsymbol{\varepsilon}}$ only and are not affected by the second-order strain $\overline{\boldsymbol{\eta}}$, as follows:

$$
\overline{\boldsymbol{\varepsilon}}=\frac{1}{V} \int_{V} \varepsilon \mathrm{d} V-\frac{1}{V} \int_{V} \operatorname{grad}[\mathbf{r}] \mathrm{d} V
$$

This relation can also be rewritten in terms of boundary integrals by applying the divergence theorem to the terms on the right-hand side of (10) to give

$$
\overline{\mathbf{\varepsilon}}=\frac{1}{V} \int_{\Gamma} \mathbf{n} \otimes \mathbf{u} \mathrm{d} \Gamma-\frac{1}{V} \int_{\Gamma} \mathbf{n} \otimes \mathbf{r} \mathrm{d} \Gamma
$$

Clearly, the volume average of the microstrain $\varepsilon$ needs to be equal to the macrostrain $\overline{\boldsymbol{\varepsilon}}$; hence, the last integral in both (10) and (11) must vanish:

$$
\int_{V} \operatorname{grad}[\mathbf{r}] \mathrm{d} V=0 \quad \text { or } \quad \int_{\Gamma} \mathbf{n} \otimes \mathbf{r} \mathrm{d} \Gamma=0
$$

Removing the term associated with $\overline{\boldsymbol{\eta}}$ from (9) by conveniently centering the RVE has the advantage that higher-order boundary conditions do not have to be prescribed on the RVE. This is consistent with the intention to preserve the microstructural RVE problem as a classical boundary value problem [2].

Following the proposed additional assumption about the second moment of area of the deformed RVE, the relation (8) is multiplied by $\mathbf{x}$ and integrated over the volume $V$ of the RVE to give

$$
\mathbf{J} \cdot \overline{\boldsymbol{\eta}}=\int_{V} \mathbf{x} \otimes \operatorname{grad}[\mathbf{u}] \mathrm{d} V-\int_{V} \mathbf{x} \otimes \operatorname{grad}[\mathbf{r}] \mathrm{d} V
$$


where $\mathbf{J}=\int_{V} \mathbf{x} \otimes \mathbf{x} \mathrm{d} V$. Once again the centering of the RVE about the macroscopic point has been utilized so that the volume integral of $\mathbf{x} \otimes \overline{\boldsymbol{\varepsilon}}$ conveniently vanishes. Applying the divergence theorem to relation (13) gives

$$
\mathbf{J} \cdot \overline{\boldsymbol{\eta}}+\frac{1}{2} \mathbf{I} \otimes \mathbf{J}: \overline{\boldsymbol{\eta}}=\int_{\Gamma} \mathbf{n} \otimes \mathbf{x} \otimes \mathbf{u} \mathrm{d} \Gamma-\int_{\Gamma} \mathbf{n} \otimes \mathbf{x} \otimes \mathbf{r} \mathrm{d} \Gamma
$$

It should be noted that the relationship between the macroscopic second-order strain (analogous to the reasoning associated with the first-order strain in (10)) and the microscopic variables is now given exclusively by integrals over the boundary of the RVE. The additional assumption regarding the second moment of area of the deformed RVE requires that the influence of the displacement fluctuation field should vanish, hence

$$
\int_{\Gamma} \mathbf{n} \otimes \mathbf{x} \otimes \mathbf{r} \mathrm{d} \Gamma=0
$$

\subsection{Upscaling}

To complete the formulation it is necessary to identify the upscaling of the microstructural response in order to define the macroscopic stress measures in terms of the microscopic quantities. For a statistically homogeneous body, the macroscopic quantities can be defined as the average of the microscopic quantities over the volume of the RVE [1]. According to the Hill-Mandel condition it is required that the local variation of work at a macroscopic point (1) must be equal to the volume average of the variation of work performed on the RVE associated with this macroscopic point:

$$
\delta \overline{\boldsymbol{\sigma}}: \overline{\boldsymbol{\varepsilon}}+\delta \bar{\tau}: \overline{\boldsymbol{\eta}}=\frac{1}{V} \int_{V} \delta \boldsymbol{\sigma}: \boldsymbol{\varepsilon} \mathrm{d} V
$$

The right-hand side of (16), for statically admissible variation of microstresses, can be expressed in terms of surface quantities (noting (7))

$$
\frac{1}{V} \int_{V} \delta \boldsymbol{\sigma}: \boldsymbol{\varepsilon} \mathrm{d} V=\frac{1}{V} \int_{\Gamma} \delta \mathbf{t} \cdot \mathbf{u} \mathrm{d} \Gamma=\frac{1}{V} \int_{\Gamma} \delta \mathbf{t} \cdot\left(\mathbf{x} \cdot \overline{\boldsymbol{\varepsilon}}+\frac{1}{2} \mathbf{x} \otimes \mathbf{x}: \overline{\boldsymbol{\eta}}+\mathbf{r}\right) \mathrm{d} \Gamma
$$

It should be noted that the work of the self-equilibrated variation of tractions $\delta \mathbf{t}$ on the rigid body translations $\mathbf{u}^{0}$ is zero; thus, the term $\mathbf{u}^{0}$ conveniently vanishes in (17).

Comparing the terms in (16) and (17), which are work conjugate to $\overline{\boldsymbol{\varepsilon}}$ and $\overline{\boldsymbol{\eta}}$ makes it possible to express the variation of the macroscopic stress quantities in terms of variation of tractions on the RVE boundary:

$$
\delta \overline{\boldsymbol{\sigma}}=\frac{1}{V} \int_{\Gamma} \mathbf{x} \otimes \delta \mathbf{t} \mathrm{d} \Gamma, \quad \delta \bar{\tau}=\frac{1}{2 V} \int_{\Gamma} \mathbf{x} \otimes \mathbf{x} \otimes \delta \mathbf{t} \mathrm{d} \Gamma
$$

Consequently, the third term in (17) needs to vanish, which places a further restriction on the displacement fluctuation field $\mathbf{r}$

$$
\int_{\Gamma} \delta \mathbf{t} \cdot \mathbf{r} \mathrm{d} \Gamma=0
$$


which together with relations (12) and (14) defines the set of boundary conditions for the RVE in an integral form as

$$
\int_{\Gamma} \delta \mathbf{t} \cdot \mathbf{r} \mathrm{d} \Gamma=0, \quad \int_{\Gamma} \mathbf{n} \otimes \mathbf{r} \mathrm{d} \Gamma=\mathbf{0}, \quad \int_{\Gamma} \mathbf{n} \otimes \mathbf{x} \otimes \mathbf{r} \mathrm{d} \Gamma=\mathbf{0}
$$

where $\delta \mathbf{t}$ represents the statically admissible variation of tractions on the boundary. The second and third integrals of (20) enforce the requirement that the effect of the displacement fluctuation field vanishes in a weak sense for a given macroscopic strain and macroscopic strain gradient.

\section{ENFORCEMENT OF THE RVE BOUNDARY CONDITIONS IN FINITE ELEMENT CONTEXT}

For the finite element analysis of the RVE, the constraint equations (20) can be restated in terms of the microscale displacement field and the macrostrain tensors (7) as

$$
\begin{array}{r}
\int_{\Gamma} \delta \mathbf{t} \cdot\left(\mathbf{u}-\mathbf{x} \cdot \overline{\boldsymbol{\varepsilon}}-\frac{1}{2} \mathbf{x} \otimes \mathbf{x}: \overline{\boldsymbol{\eta}}\right) \mathrm{d} \Gamma=0 \\
\int_{\Gamma} \mathbf{n} \otimes\left(\mathbf{u}-\mathbf{x} \cdot \overline{\boldsymbol{\varepsilon}}-\frac{1}{2} \mathbf{x} \otimes \mathbf{x}: \overline{\boldsymbol{\eta}}\right) \mathrm{d} \Gamma=\mathbf{0} \\
\int_{\Gamma} \mathbf{n} \otimes \mathbf{x} \otimes\left(\mathbf{u}-\mathbf{x} \cdot \overline{\boldsymbol{\varepsilon}}-\frac{1}{2} \mathbf{x} \otimes \mathbf{x}: \overline{\boldsymbol{\eta}}\right) \mathrm{d} \Gamma=\mathbf{0}
\end{array}
$$

Although these conditions can be applied in a variety of ways, typically (21) is enforced directly, whereas (22)-(23) are a priori satisfied by the choice of traction, periodic or displacement boundary conditions.

\subsection{Boundary conditions in matrix form}

Switching now to matrix-vector notation, after FE discretization of the RVE, the boundary conditions (21)-(23) can be written in a matrix form as

$$
\mathbf{C u}=\mathbf{D} \overline{\boldsymbol{\varepsilon}}+\mathbf{E} \overline{\boldsymbol{\eta}}=\mathbf{g}
$$

where matrices $\mathbf{C}, \mathbf{D}$, and $\mathbf{E}$ are given by

$$
\mathbf{C}=\int_{\Gamma} \mathbf{H} \mathbf{N}^{\mathrm{T}} \mathbf{N} \mathrm{d} \Gamma, \quad \mathbf{D}=\int_{\Gamma} \mathbf{H} \mathbf{N}^{\mathrm{T}} \mathbf{X} \mathrm{d} \Gamma, \quad \mathbf{E}=\int_{\Gamma} \mathbf{H} \mathbf{N}^{\mathrm{T}} \mathbf{Z} \mathrm{d} \Gamma
$$

$\mathbf{N}$ is the matrix of shape functions and matrices $\mathbf{X}$ and $\mathbf{Z}$ are defined as

$$
\mathbf{X}=\frac{1}{2}\left[\begin{array}{ccc}
2 x & 0 & y \\
0 & 2 y & x
\end{array}\right], \quad \mathbf{Z}=\frac{1}{4}\left[\begin{array}{cccccc}
2 x^{2} & 0 & 2 y^{2} & 0 & x y & 0 \\
0 & 2 y^{2} & 0 & 2 x^{2} & 0 & x y
\end{array}\right]
$$

This approach is restricted here to the $2 \mathrm{D}$ case but can be easily extended to $3 \mathrm{D}$. 


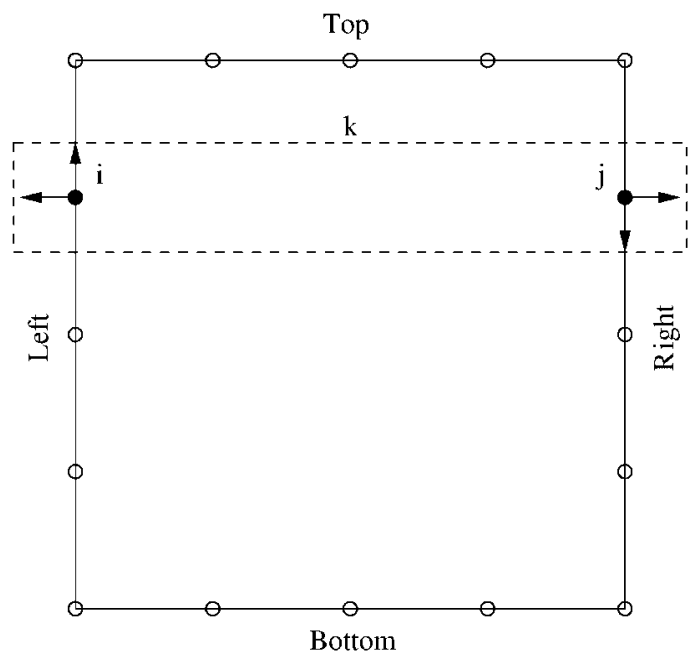

Figure 2. Nodal values of weight functions corresponding to antiperiodic tractions at nodes $i$ and $j$ for periodic boundary conditions applied to a square RVE.

Relation (24) is valid for a generalized form of the RVE boundary conditions and the specific nature of the RVE boundary conditions (i.e. displacement, periodic, or traction) is reflected by different terms of the corresponding $\mathbf{H}$ matrix. Each row of the matrix $\mathbf{H}$ represents an admissible distribution of nodal traction forces on the boundary of the RVE. Accordingly, the boundary condition for the RVE is multiplied by the weight functions in matrix $\mathbf{H}$ and integrated over the boundary to give relation (24).

In the case of periodic boundary conditions, matrix $\mathbf{H}$ contains nodal values of all linearly independent antiperiodic distribution of tractions on the boundary of the RVE. Restricting ourselves for the moment to first-order computational homogenization, Figure 2 shows the boundary nodes of a quadrilateral RVE, highlighting nodes $i$ and $j$ on opposite sides of the RVE as well as arrows representing the weight functions for antiperiodic tractions as appropriate. The associated $\mathbf{H}$ matrix is as follows:

$$
\mathbf{H}=\left[\begin{array}{ccccccccc}
\ldots & \ldots & \ldots & \ldots & \ldots & \ldots & \ldots & \ldots & \ldots \\
0 & \ldots & -1 & 0 & \ldots & 1 & 0 & \ldots & 0 \\
0 & \ldots & 0 & 1 & \ldots & 0 & -1 & \ldots & 0 \\
\ldots & \ldots & \ldots & \ldots & \ldots & \ldots & \ldots & \ldots
\end{array}\right]
$$

Subsequent rows in matrix $\mathbf{H}$ lead to multiple constraints which connect degrees of freedom on the left and right sides as well as connect degrees of freedom on the top and bottom sides of the RVE's boundary.

We note that for periodic boundary conditions in the second-order computational homogenization, the RVE deforms according to the first-order strain $\bar{\varepsilon}$ and only to the symmetric part of the second-order strain $\overline{\boldsymbol{\eta}}[2]$. To enforce the deformation of the RVE corresponding to the 

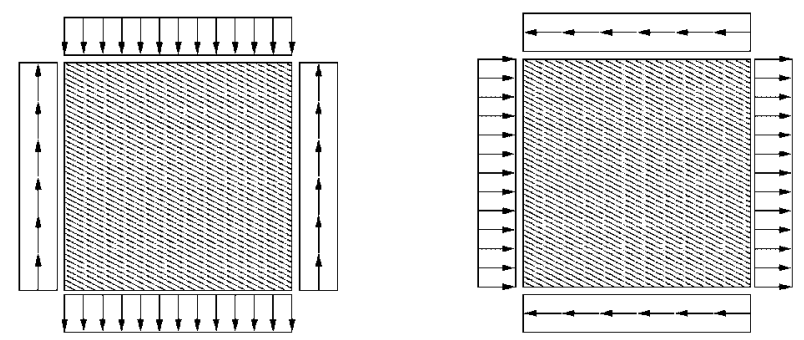

Figure 3. Distribution of admissible tractions for periodic boundary conditions which allow deformation of the RVE according to the antisymmetric part of second-order strain $\bar{\eta}$.
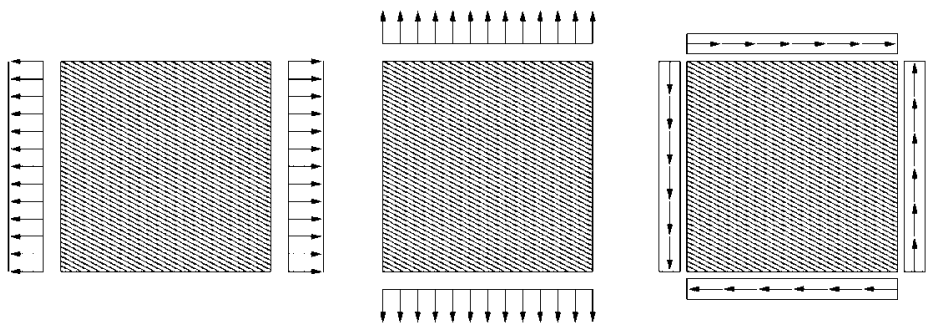

Figure 4. Distribution of admissible tractions for traction boundary conditions which lead to the deformation of RVE according to first-order strain $\varepsilon$.
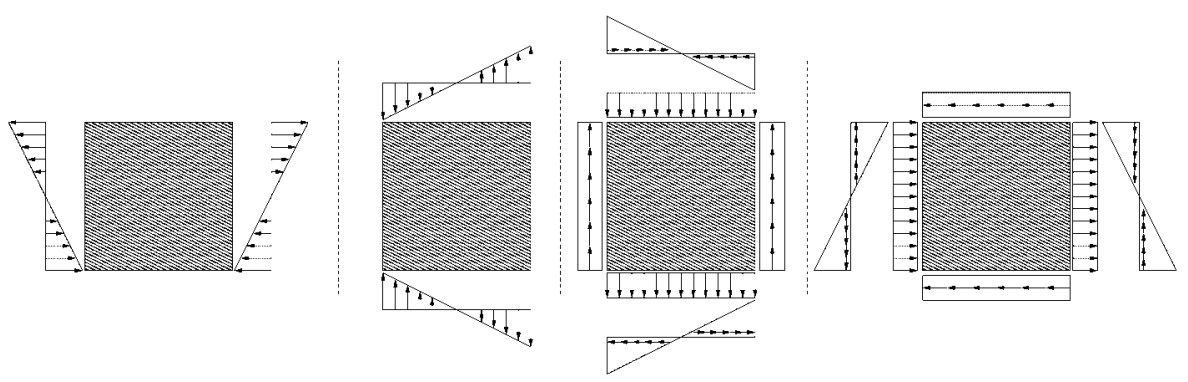

Figure 5. Distribution of admissible tractions for traction boundary conditions which lead to the deformation of RVE according to second-order strain $\boldsymbol{\eta}$.

antisymmetric part of $\overline{\boldsymbol{\eta}}$, additional constraints have to be added. Consequently, the $\mathbf{H}$ matrix is augmented to include the admissible distribution of tractions as shown in Figure 3.

In the case of traction boundary conditions, the admissible distribution of tractions corresponding to $\overline{\boldsymbol{\varepsilon}}$ is shown in Figure 4 and for $\overline{\boldsymbol{\eta}}$ in Figure 5.

In the case of displacement boundary conditions, the $\mathbf{H}$ matrix is simply the identity matrix. Thus, depending on the type of boundary condition, only the $\mathbf{H}$ matrix changes. This approach allows the enforcement of generalized boundary conditions to the RVE. It is also worth noting that this method can easily be applied to an arbitrary shape of the RVE (although we restrict ourselves 
to quadrilaterals in this work). For completeness, it should be noted that the removal of the rigid body modes is readily achieved by augmenting the $\mathbf{H}$ matrix with the appropriate constraints.

\subsection{Enforcement of boundary conditions (multiple constraints)}

The solution of the RVE boundary value problem can be expressed as a constrained quadratic programming problem:

$$
\begin{aligned}
& \min _{\mathbf{u}} \quad \mathscr{Q}=\frac{1}{2} \mathbf{u}^{\mathrm{T}} \mathbf{K u}-\mathbf{u}^{\mathrm{T}} \mathbf{F} \\
& \text { subject to } \mathbf{C u}-\mathbf{g}=\mathbf{0}
\end{aligned}
$$

where $\mathbf{K}$ is the stiffness matrix, $\mathbf{F}$ is the load vector, $\mathbf{C}$ is the constraint matrix, and $\mathbf{g}$ is the displacement constraint vector. A common method to solve this problem is to introduce Lagrange multipliers $\lambda$ :

$$
\min _{\mathbf{u}, \mathscr{L}} \mathscr{L}=\frac{1}{2} \mathbf{u}^{\mathrm{T}} \mathbf{K} \mathbf{u}-\mathbf{u}^{\mathrm{T}} \mathbf{F}+\lambda^{\mathrm{T}}(\mathbf{C u}-\mathbf{g})
$$

The Euler conditions for the stationary point of the Lagrangian are found to be

$$
\begin{aligned}
\mathbf{K u}+\mathbf{C}^{\mathrm{T}} \lambda & =\mathbf{F} \\
\mathbf{C u} & =\mathbf{g}
\end{aligned}
$$

However, this approach increases the number of unknowns and the character of the system matrix is altered (to an indefinite saddle point problem). Moreover, the numerical solution is rather inefficient and therefore not suitable for solving computationally complex multi-scale problems where the constrained quadratic problem has to be solved for every integration point. Another common approach is the imposition of the RVE boundary conditions by static condensation (for example $[9,10])$; however, the computational procedure for this approach is explicitly linked to the nature of the boundary conditions and it differs depending on whether periodic, displacement, or traction boundary conditions are applied. The novel unified approach to formulate the generalized boundary conditions for the RVE advocated in Section 4.1 allows for a different approach following the work of Ainsworth [5] for multiple constraints to be adopted here. Assuming that the problem (28) is well posed, the following matrices are well defined:

$$
\mathbf{Q}=\mathbf{I}-\mathbf{R C} \quad \text { and } \quad \mathbf{R}=\mathbf{C}^{\mathrm{T}}\left(\mathbf{C} \mathbf{C}^{\mathrm{T}}\right)^{-1}
$$

where $\mathbf{R}$ is an auxiliary matrix, $\mathbf{Q}$ is a projection matrix, and $\mathbf{C}$ is the constraint matrix from the original equation (28). If the matrix $\widetilde{\mathbf{K}}$ and the right hand vector $\widetilde{\mathbf{F}}$ are defined by the following expressions:

$$
\begin{aligned}
& \widetilde{\mathbf{K}}=\mathbf{C}^{\mathrm{T}} \mathbf{C}+\mathbf{Q}^{\mathrm{T}} \mathbf{K} \mathbf{Q} \\
& \widetilde{\mathbf{F}}=\mathbf{C}^{\mathrm{T}} \mathbf{g}+\mathbf{Q}^{\mathrm{T}}(\mathbf{F}-\mathbf{K} \mathbf{R g})
\end{aligned}
$$

there exists a unique solution $\mathbf{u}$ to the problem (28)

$$
\mathbf{u}=\widetilde{\mathbf{K}}^{-1} \widetilde{\mathbf{F}}
$$


and the corresponding Lagrange multipliers can be recovered from

$$
\lambda=\mathbf{R}^{\mathrm{T}}(\mathbf{F}-\mathbf{K u})
$$

Furthermore, a significant advantage of the proposed approach is that in practical computations there is no need to perform global operations on matrices or to assemble the global matrix K. Enforcing constraints can be performed via an element-by-element subassembly procedure [5].

\subsection{Computation of the macroscopic stress and higher-order stress}

Given that the solution for $\mathbf{u}$ (from (34)) and $\lambda$ (from (35)) satisfies the equilibrium, the work done by the tractions on the displacements is equal to the work of the generalized tractions (Lagrange multipliers) on the generalized displacements (strains and second-order strains):

$$
\mathbf{u}^{\mathrm{T}} \mathbf{t}=(\mathbf{D} \overline{\boldsymbol{\varepsilon}}+\mathbf{E} \overline{\boldsymbol{\eta}})^{\mathrm{T}} \boldsymbol{\lambda}
$$

After the solution of the RVE boundary value problem it is necessary to determine the macroscopic stress and higher-order stress quantities. With reference to (18), the first- and second-order macrostress vectors can be expressed in terms of the Lagrange multipliers $\lambda$ and matrices $\mathbf{D}$ and $\mathbf{E}$ :

$$
\overline{\boldsymbol{\sigma}}=\frac{1}{V} \mathbf{D}^{\mathrm{T}} \lambda, \quad \bar{\tau}=\frac{1}{V} \mathbf{E}^{\mathrm{T}} \boldsymbol{\lambda}
$$

\subsection{Computation of tangent matrices}

In computational homogenization, the stress-strain relationship at the macro-level is not known a priori. For the finite element analysis to proceed at the macro-level both the stress state (37) and the material tangent stiffness matrices have to be determined at each integration point. The linearized relation between strain increments and stress increments for the second-order continuum can be written as

$$
\begin{aligned}
\Delta \overline{\boldsymbol{\sigma}} & =\overline{\mathbf{C}}^{1} \Delta \overline{\boldsymbol{\varepsilon}}+\overline{\mathbf{C}}^{2} \Delta \overline{\boldsymbol{\eta}} \\
\Delta \bar{\tau} & =\overline{\mathbf{C}}^{3} \Delta \overline{\boldsymbol{\varepsilon}}+\overline{\mathbf{C}}^{4} \Delta \overline{\boldsymbol{\eta}}
\end{aligned}
$$

In order to compute the terms of the tangent stiffness matrices, it is necessary to solve $(3+6+$ $3+6=18$ ) linear equations for each RVE, associated with incremental changes of macroscopic strains. For example the material tangent stiffness matrix $\overline{\mathbf{C}}^{1}$ is computed as

$$
\overline{\mathbf{C}}^{1}=\left[\delta \overline{\boldsymbol{\sigma}}^{1}, \delta \overline{\boldsymbol{\sigma}}^{2}, \delta \overline{\boldsymbol{\sigma}}^{3}\right]
$$

where columns $\delta \overline{\boldsymbol{\sigma}}^{i}, i=1,2,3$, are computed for given increments of the strain vectors

$$
\begin{array}{ll}
\delta \overline{\boldsymbol{\sigma}}^{1}: & \text { for } \delta \overline{\boldsymbol{\varepsilon}}=\left[\begin{array}{lll}
1 & 0 & 0
\end{array}\right]^{\mathrm{T}}, \delta \overline{\boldsymbol{\eta}}=\left[\begin{array}{lllll}
0 & 0 & 0 & 0 & 0
\end{array}\right]^{\mathrm{T}} \\
\delta \overline{\boldsymbol{\sigma}}^{2}: & \text { for } \delta \overline{\boldsymbol{\varepsilon}}=\left[\begin{array}{lll}
0 & 1 & 0
\end{array}\right]^{\mathrm{T}}, \delta \overline{\boldsymbol{\eta}}=\left[\begin{array}{llllll}
0 & 0 & 0 & 0 & 0 & 0
\end{array}\right]^{\mathrm{T}} \\
\delta \overline{\boldsymbol{\sigma}}^{3}: & \text { for } \delta \overline{\boldsymbol{\varepsilon}}=\left[\begin{array}{lll}
0 & 0 & 1
\end{array}\right]^{\mathrm{T}}, \delta \overline{\boldsymbol{\eta}}=\left[\begin{array}{llllll}
0 & 0 & 0 & 0 & 0 & 0
\end{array}\right]^{\mathrm{T}}
\end{array}
$$


It should be noted that for each of these cases only the right-hand side of the linear equation system differs; hence, the computation of tangent stiffness matrices is equivalent to multiple load cases and can be undertaken relatively efficiently.

\subsection{Analytical validation}

Further insight and validation of the computational homogenization scheme can be provided by considering the limiting case of a homogeneous non-local Hooke's material similar to the discussion provided in [10]. For such a material, the result of homogenization should not depend on the type of the RVE boundary conditions. The tangent material matrix $\overline{\mathbf{C}}^{1}$ is given as

$$
\left[\begin{array}{l}
\bar{\sigma}_{11} \\
\bar{\sigma}_{22} \\
\bar{\sigma}_{12}
\end{array}\right]=\left[\begin{array}{ccc}
\lambda+2 \mu & \lambda & 0 \\
\lambda & \lambda+2 \mu & 0 \\
0 & 0 & 2 \mu
\end{array}\right]\left[\begin{array}{c}
\bar{\varepsilon}_{11} \\
\bar{\varepsilon}_{22} \\
2 \bar{\varepsilon}_{12}
\end{array}\right]
$$

It can be noted that the solution depends only on material parameters $\lambda$ and $\mu$, since for a classical continuum we assume that the intrinsic length size is infinitesimally small compared with the characteristic size of a structure at the macroscale of observation. An exact solution for the terms of the higher-order tangent matrix $\overline{\mathbf{C}}^{4}$ for periodic and traction boundary conditions can be determined using a variety of analysis techniques. In the finite element context, the use of a nine-noded Lagrangian element is required to ensure that the stress field satisfies equilibrium in a pointwise sense:

$$
\left[\begin{array}{c}
\bar{\tau}_{111} \\
\bar{\tau}_{222} \\
\bar{\tau}_{221} \\
\bar{\tau}_{112} \\
\bar{\tau}_{121} \\
\bar{\tau}_{122}
\end{array}\right]=d\left[\begin{array}{cccccc}
a & 0 & -a & 0 & 0 & -b \\
0 & a & 0 & -a & -b & 0 \\
-a & 0 & a & 0 & 0 & b \\
0 & -a & 0 & a & b & 0 \\
0 & -b & 0 & b & c & 0 \\
-b & 0 & b & 0 & 0 & c
\end{array}\right]\left[\begin{array}{c}
\bar{\eta}_{111} \\
\bar{\eta}_{222} \\
\bar{\eta}_{221} \\
\bar{\eta}_{112} \\
2 \bar{\eta}_{121} \\
2 \bar{\eta}_{122}
\end{array}\right]
$$

where

$$
a=L^{2}(2 \mu+\lambda) / 2, \quad b=L^{2} \mu, \quad c=L^{2}(3 \mu+\lambda), \quad d=\frac{\mu}{3(4 \mu+\lambda)}
$$

We note that the higher-order tangent matrix $\overline{\mathbf{C}}^{4}$ for the second-order continuum depends on the edge length $L$ of the RVE considered; thus, higher-order homogenization approaches are able to take into account size effects. Owing to the material homogeneity, material isotropy, and symmetry of the RVE for the model problem considered, the higher-order tangents $\overline{\mathbf{C}}^{2}$ and $\overline{\mathbf{C}}^{3}$ are both zero.

The proposed second-order computational homogenization scheme recovers the expected $\overline{\mathbf{C}}^{1}$ constitutive matrix in all cases. The terms of the constitutive matrix $\overline{\mathbf{C}}^{4}$ are recovered for periodic or traction boundary conditions, but in the case of displacement boundary conditions the terms of $\overline{\mathbf{C}}^{4}$ differ. This apparent inconsistency (as the terms of the $\overline{\mathbf{C}}^{4}$ matrix should not be affected by the choice of the RVE boundary conditions) is inconsequential since, for any statically admissible second-order strain $\overline{\boldsymbol{\eta}}$, the displacement fluctuation field in the RVE vanishes and the second-order 
stress $\bar{\tau}$ for the displacement boundary conditions is equal to the second-order stress obtained by the application of periodic and traction boundary conditions. Thus, in an asymptotic sense, the results from homogenization for general heterogeneous materials should not be affected by the choice of the RVE boundary conditions.

\section{NUMERICAL EXAMPLE: SHEAR LAYER PROBLEM}

In this section a boundary shear layer problem is investigated assuming a microstructurally porous material. A second-order computational homogenization scheme as advocated in Section 3 is adopted for all analyses. The macroscale mesh comprises plane strain quadrilateral elements for the second-order continua (QU34L4 element). Since the problem is independent of $x$, only a single column of elements is needed, see Figure 6. The problem is analysed for two different heights: $H=10.0$ and $1.0 \mathrm{~mm}$.

The material comprises a linear elastic matrix with randomly distributed voids. For modelling the microscale two different types of RVE (see Figure 7 for representative microstructures) of length $L=0.6 \mathrm{~mm}$ and a fixed void ratio of 0.12 are considered; these comprise randomly distributed

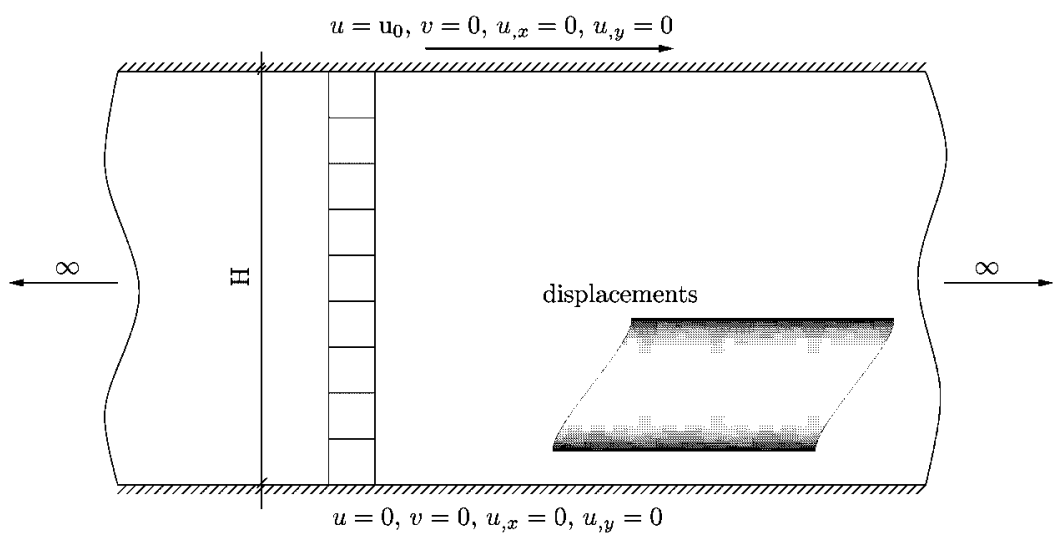

Figure 6. Geometry, boundary conditions and finite element mesh for shear layer problem.

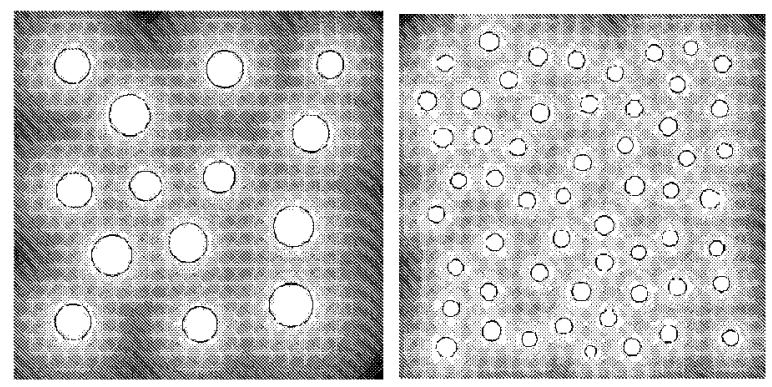

Figure 7. RVEs comprising voids with average radii $r_{\mathrm{ave}}=0.032$ and $0.016 \mathrm{~mm}$. Contours of 0 -order level set function. 

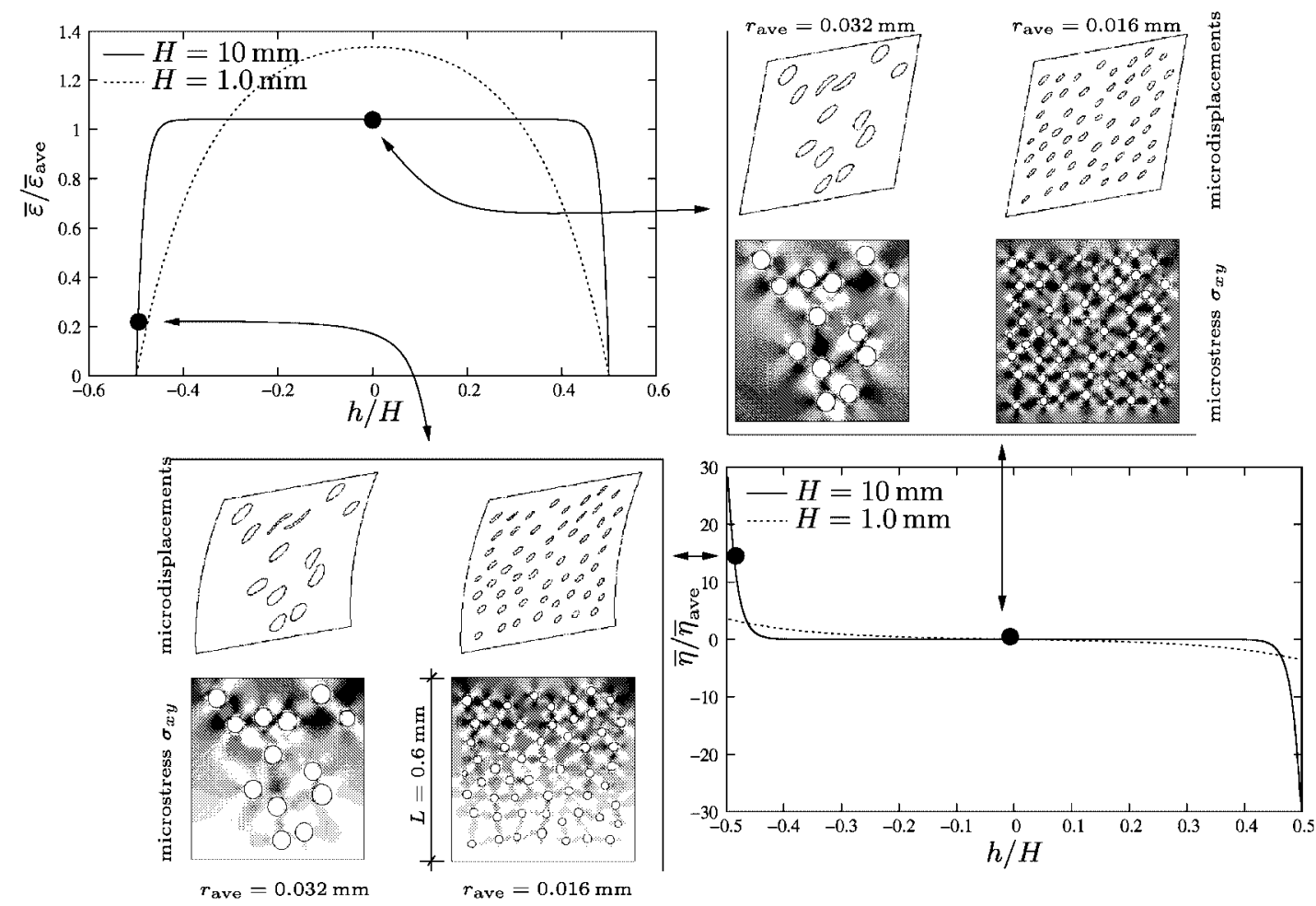

Figure 8. Graphs of shear strain and gradient of shear strain through the height of the shear layer for mesh heights of 10 and $1 \mathrm{~mm}$ and for RVEs with both small and large voids. Deformation and the distribution of the shear microstress of typical RVEs at the middle and bottom of the shear layer are also shown.

voids with different average radii $\left(r_{\mathrm{ave}}=0.032\right.$ and $\left.0.016 \mathrm{~mm}\right)$. Voids are modelled by the level set method (within the context of the extended finite element method (XFEM) [11]). The finite element mesh of the RVE comprises constant strain triangular elements with a characteristic size $h^{e}=0.008 \mathrm{~mm}$. Young's modulus of the matrix is $E=210 \mathrm{GPa}$ and Poisson ratio $v=0.3$. In order to investigate the influence of the microstructure, 100 randomly generated microstructures (using a normal distribution with a standard deviation of $0.002 \mathrm{~mm}$ ) are considered for both types of RVE (small and large voids) and for each height of the shear layer.

The distribution of shear strain and gradient of shear strain through the height of the shear layer is shown in Figure 8. These results illustrate that the solution depends on the height of the shear layer and that size effect is observed. It is also worth noting that near the upper and lower boundaries, gradients of shear strain are clearly observed. It is clear that if the macroscopic level had been modelled using a classical continuum these boundary effects would not have been evident and the shear strain and shear stress would have been predicted as constant through the height of the shear layer. Figure 8 also shows the deformation of typical RVEs in the centre and at the bottom of the shear layer as well as the shear microstress distribution. A gradient in the shear microstress across the height of those RVEs located at the bottom of the shear layer is clearly observed, whereas no such gradient exists for RVEs located in the middle of the shear layer, corresponding to pure shear. 

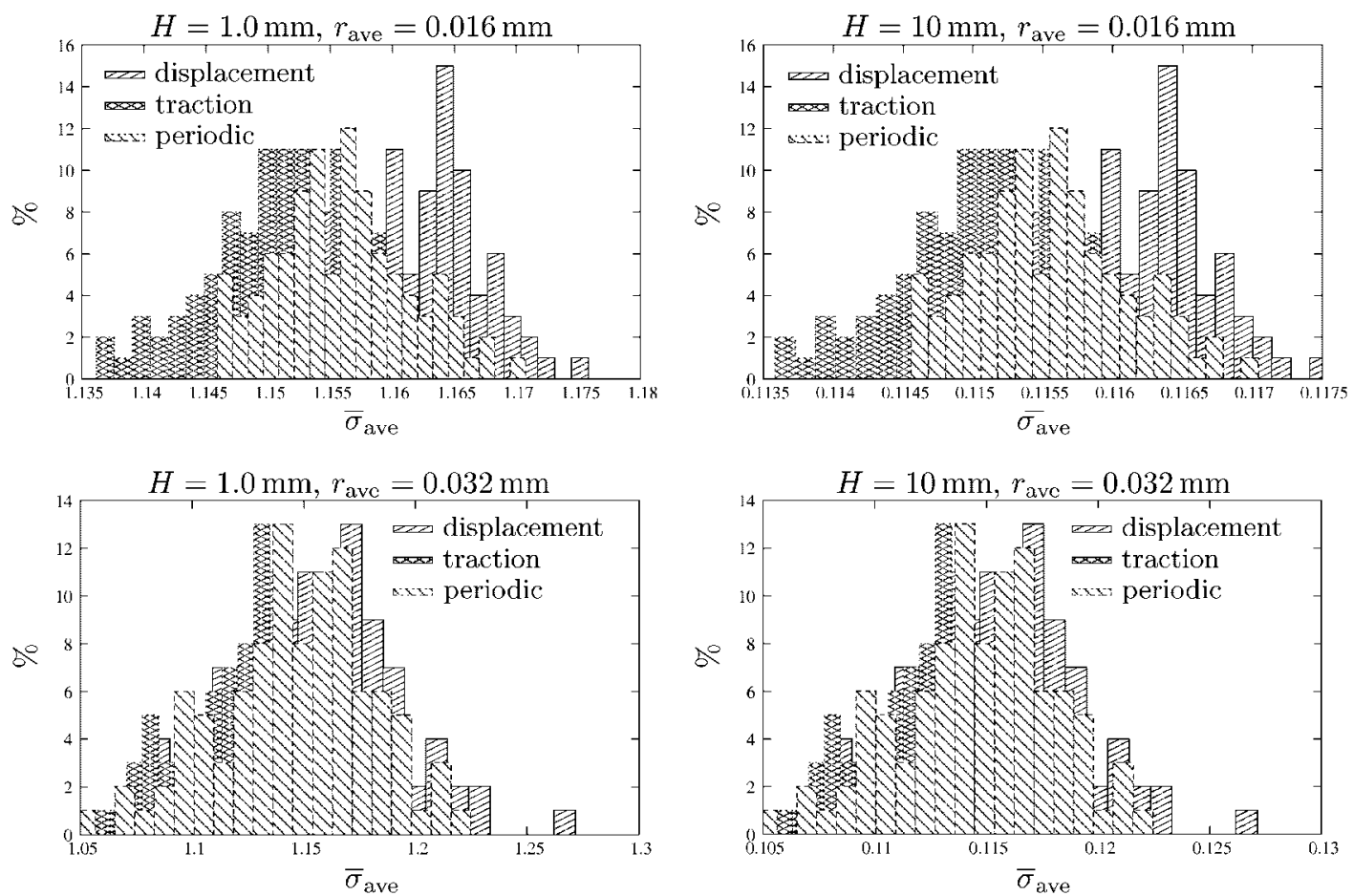

Figure 9. Histogram for average values of shear stress for displacement, periodic, and traction boundary conditions for shear layer problem (two heights of layer and two average void sizes).
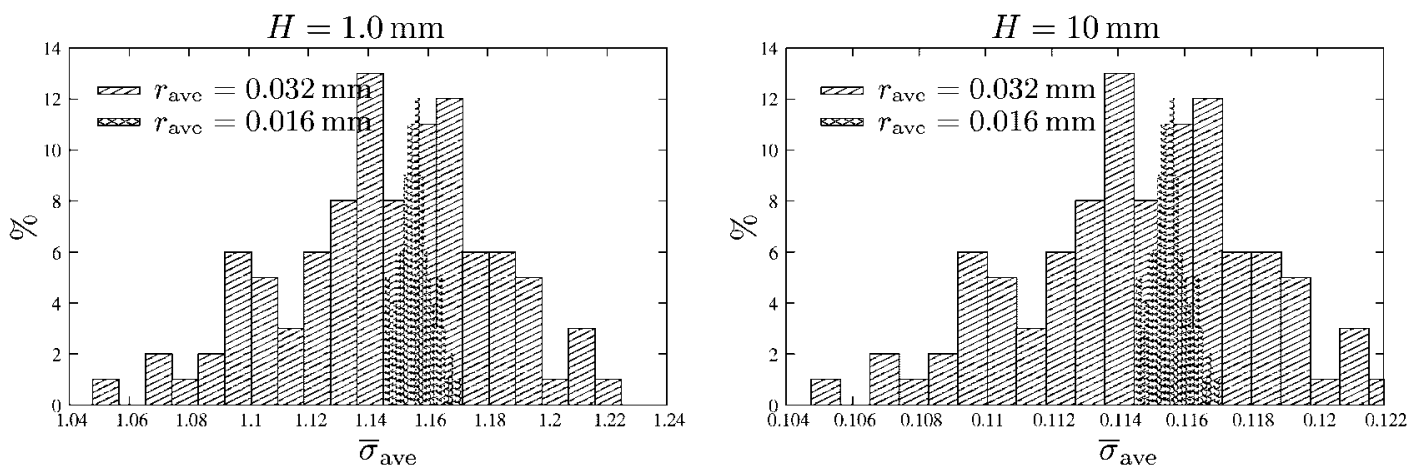

Figure 10. Histogram for averaged values of shear stress for periodic boundary conditions for shear layer problem (two average heights of layer and two average void sizes).

It is often argued that the size of the RVE should be selected as the minimum size that contains sufficient microstructural features to be statistically representative of the material. However, the absolute size of the RVE (intrinsic length scale) is also an additional macroscopic parameter which, unlike the constitutive relationship, cannot be determined via the homogenization method. Once 
the size of the RVE has been assumed, the statistical representation can be determined via a number of microstructural random realizations and measured as the difference between the upper and lower bounds of the solution. Figure 9 shows histograms of the average macroscopic shear stress within the shear layer for the two different heights considered and for the two different radii of voids in the RVE. The results illustrate (and hence confirm the tacit assumption made by other researchers) that the displacement and traction RVE boundary conditions provide the upper and lower bounds of the response and the solution associated with the periodic RVE boundary conditions lies between them.

The responses associated with RVEs with a small void radius have a smaller variation and are thus more statistically representative than those with a larger void radius. In the case of periodic boundary conditions, a smaller variance in the solutions is observed (Figure 10). It can also be seen that the solutions for different average void radii converge to the same value.

\section{CONCLUSIONS}

This paper considers the formulation of the equations coupling the microscopic and macroscopic variables in second-order computational homogenization and the definition and enforcement of boundary conditions for the RVE. The proposed formulation is deliberately general so that any type of RVE boundary conditions (e.g. displacement, traction, periodic) can be applied in a unified manner. The construction of the boundary conditions has been decoupled from the method by which they are numerically enforced. This numerical enforcement has been undertaken using projection matrices for multiple constraints in a simple and efficient manner.

The numerical example compares results for different boundary conditions for the second-order scheme, as well as investigates how the nature of the microstructure affects the macroscopic response. The commonly held assumption that displacement and traction boundary conditions provide an upper- and lower-bound solution has been confirmed for second-order computational homogenization.

\section{ACKNOWLEDGEMENTS}

The authors gratefully acknowledge the support of the U.K. Engineering and Physical Sciences Research Council (Grant Ref: EP/D500273). The first author also acknowledges the support of the Polish Ministry of Scientific Research and Information Technology Society (Grant Ref: 4 T07E060 29), and guidance received from Professor Zenon Waszczyszyn of Cracow University of Technology.

\section{REFERENCES}

1. Nemat-Nasser S, Hori M. Micromechanics: Overall Properties of Heterogeneous Materials. Elsevier: Amsterdam, 1999.

2. Kouznetsova VG. Computational homogenization for the multi-scale analysis of multi-phase materials. Ph.D. Thesis, Technische Universiteit, Eindhoven, 2002.

3. Feyel F. Multiscale FE2 elastoviscoplastic analysis of composite structures. Computational Materials Science 1999; 16:344-354.

4. Feyel F. A multilevel finite element method (FE2) to describe the response of highly non-linear structures using generalized continua. Computer Methods in Applied Mechanics and Engineering 2003; 192:3233-3244.

5. Ainsworth M. Essential boundary conditions and multi-point constraints in finite element analysis. Computer Methods in Applied Mechanics and Engineering 2001; 190:6323-6339. 
6. Toupin RA. Elastic materials with couple-stresses. Archives Rational Mechanical Analysis 1968; 11:385-413.

7. Mindlin RD. Second gradient of strain and surface-tension in linear elasticity. International Journal of Solids and Structures 1965; 1:417-438.

8. Shu JY, King WE, Fleck NA. Finite element for materials with strain gradient effects. International Journal for Numerical Methods in Engineering 1999; 44(1999):373-391.

9. Miehe C, Koch A. Computational micro-to-macro transitions of discretized microstructures undergoing small strains. Archive of Applied Mechanics 2002; 72:300-317.

10. Kouznetsova VG, Geers MGD, Brekelmans WAM. Size of a representative volume element in a second-order computational homogenization framework. International Journal for Multiscale Computational Engineering 2004; 2(4):575-598.

11. Belytschko T, Moës N, Usui S, Parimi C. Arbitrary discontinuities in finite elements. International Journal for Numerical Methods in Engineering 2001; 50:993-1013. 